\title{
Design and development of a slot-less permanent magnet linear motor using permeance analysis method for spray application
}

\begin{abstract}
Mostly in industrial spray applications pneumatic systems are utilized for operating the automatic spray gun. Linear motor can be one of the alternatives for triggering the spray gun instead of pneumatic systems due to its accuracy in valve positioning according to the required flow rate. From this point of view a tubular linear permanent magnet motor has been designed using Permeance Analysis Method (PAM) and developed. Three permeance models have been developed for PAM analysis. Among these three models, only one model is selected as a PAM model which can be produced the required amount of thrust for triggering the spray gun. After selecting the PAM thrust model, the size of the motor has been optimized by analyzing the effect of thrust constant, electrical and mechanical time constant. Finally based on the optimized data, the motor has been fabricated and tested that shows the good argument with the analysis result.
\end{abstract}

Keyword: Linear motor; Thrust constant; Electrical time constant; Mechanical time constant 